\section{POS0091 OFF-TARGET PROFILING OF JANUS KINASE (JAK) INHIBITORS IN RHEUMATOID ARTHRITIS: A COMPUTER-BASED APPROACH FOR DRUG SAFETY STUDIES AND REPURPOSING}

M. L. Faquetti ${ }^{1}$, F. Grisoni ${ }^{1}$, P. Schneider ${ }^{2}$, G. Schneider ${ }^{2}$, A. M. Burden ${ }^{1} .{ }^{1} E T H$ Zurich, Department of Chemistry and Applied Biosciences, Zürich, Switzerland; ${ }^{1}$ ETH Zurich, Department of Chemistry and Applied Biosciences, Zürich, Switzerland

Background: The JAK inhibitors (JAKi's) tofacitinib and baricitinib are new alternatives for treating rheumatoid arthritis. Safety concerns associated with JAKi's, such as the increased risk for thrombosis and viral infections, have emerged worldwide. The underlying explanatory mechanisms remain unknown, suggesting the elevated risk is likely due to underlying confounding or an off-target binding effect. Computational approaches can explore the potential for a small molecule drug to interact with previously unknown biological targets and identify potential safety-related concerns, and open doors for potential drug repurposing Objectives: To identify and characterize the off-target binding effects of baricitinib and tofacitinib, with a focus on targets related to thrombosis and viral infection Methods: Potential targets of baricitinib and tofacitinib were predicted using two neural-network-based systems (TIGER[1] and SPiDER[2]). Targets were considered relevant if they had (1) a SPiDER confidence with $p<0.05$, or (2) a TIGER score $>1$. Selected targets related to the outcome of interest were experimentally evaluated at Eurofins Cerep (France-Celle L'Evescault, www.eurofins. com) if commercial available. Compounds were tested at (1) single concentration $(30 \mu \mathrm{M})$ with technical replicates, using radioligand or enzymatic assays, or (2) multiple concentrations ( $30 \mu \mathrm{M}$ highest concentration; dilution factor in a logscale) with technical replicates, using calcium flux or inhibition of [cAMP] assays. Observed activity of $\geq 50 \%$ inhibition or stimulation on the target was considered active, between 25 to $50 \%$ inhibition (or a dissociation constant [Kd] from 1 to 10 $\mu \mathrm{M})$ was considered as moderate activity, and lower than $25 \%$ was considered inactive. Dose-response curve were performed on active and moderate targets for IC ${ }_{50} / \mathrm{EC}_{50}$ (half maximal inhibitory / effective concentration) determination. Results: TIGER and SPIDER suggested a total of 99 off-target binding effects (baricitinib $n=41$; tofacitinib $n=58$ ), of which 17 targets had potential impact on thrombosis or viral infection (baricitinib $n=5$ and 4 , respectively; tofacitinib $n=5$ and 3, respectively). Commercial testing was available on 11 targets (Adenosine Receptor A2A [AA2AR], Epidermal growth factor receptor, induclible NOS, PI3 Kinase (p110b/p85a), Phosphodiesterase 10A2 [PDE10A2] and Protein Kinase N2 [PKN2] for baricitinib; and Adenosine receptor A3, 15-Lipoxygenase [15-LO], PKN2, Transient receptor potential cation channel [TRPM6] and AA2AR for tofacitinib). Of these, 5 targets showed active or moderately active binding activity (baricitinib $n=2$; tofacitinib $n=3$ ), and were tested for dose-response curves. Test results confirmed ligand-binding activity with $\mathrm{IC}_{50}$ on nanomolar (PKN2), and micromolar ranges (PDE10A2 and TRPM6).

Conclusion: The results suggest both baricitinib and tofacitinib are promiscuous binders with effects on several families. Although it may lead to side effects, off-target binding also represents a potential opportunity for drug repurposing. Besides on-target effects, both drugs are under clinical investigation for the treatment of COVID19 due to off-target interactions. The proposed pharmacological off-target effects of those with active binding include attenuation of pulmonary vascular remodeling, anti-fibrotic and anti-psychotic activities (PDE10A2), modulation of viral response (PKN2), and hypomagnesaemia (TRPM6), which is involved in cardiovascular diseases. This study supports tofacitinib and baricitinib as candidates for drug repurposing (e.g., in COVID-19, Hepatitis C virus, and pulmonary hypertension). We did not identify active off-target interactions linked to thrombosis to explain the elevated risk observed in clinical practice. Further research is required to elucidate the underlying patient-specific factors (confounders) that could explain this safety concern. REFERENCES

[1] Schneider P et al. Angew Chem Int Ed 2017;56:11520-4.

[2] Reker D et al. PNAS 2014;111:4067-72.

Disclosure of Interests: None declared

DOI: 10.1136/annrheumdis-2021-eular.982

\section{POS0092 \\ HERPES ZOSTER IN THE FILGOTINIB RHEUMATOID ARTHRITIS PROGRAM}

K. Winthrop ${ }^{1}$, M. H. Buch ${ }^{2}$, J. Curtis ${ }^{3}$, G. R. Burmester ${ }^{4}$, D. Aletaha ${ }^{5}$, K. Amano ${ }^{6}$ A. Pechonkina ${ }^{7}$, I. Tiamiyu ${ }^{8}$, C. Leatherwood ${ }^{9}$, L. Y $\mathrm{Y}^{10}, \mathrm{Q}$. Gong ${ }^{10}$, R. Besuyen ${ }^{11}$ J. Galloway ${ }^{12} .{ }^{1}$ Oregon Health and Science University, Division of Infectious Diseases, Portland, United States of America; ${ }^{2}$ University of Manchester, Division of Musculoskeletal \& Dermatological Sciences, Manchester, United Kingdom; ${ }^{3}$ University of Alabama at Birmingham, Division of Clinical Immunology \& Rheumatology, Birmingham, United States of America; ${ }^{4}$ Charité University Hospital Berlin, Department of Rheumatology and Clinical Immunology, Berlin, Germany; ${ }^{5}$ Medical University of Vienna, Division of Rheumatology, Department of Medicine 3, Vienna, Austria; ${ }^{6}$ Saitama Medical University, Department of Rheumatology and Clinical Immunology, Saitama, Japan; ${ }^{7}$ Gilead Sciences, Inc., Inflammation and Respiratory Therapeutic Area, Foster City, United States of America; ${ }^{8}$ Gilead Sciences, Inc., Clinical Research, Foster City, United States of America; ${ }^{9}$ Gilead Sciences, Inc., Inflammation \& Respiratory Therapeutics, Foster City, United States of America; ${ }^{10}$ Gilead Sciences, Inc., Biostatistics, Foster City, United States of America; ${ }^{11}$ Galapagos BV, Clinical Development, Leiden, Netherlands; ${ }^{12}$ King's College London, Centre for Rheumatic Diseases, School of Immunology and Microbial Sciences, London, United Kingdom

Background: The once daily, oral Janus kinase (JAK)-1 preferential inhibitor filgotinib (FIL) improved signs and symptoms of rheumatoid arthritis (RA) in phase $(\mathrm{P}) 3$ trials. ${ }^{1-3}$ Patients (pts) with RA have increased herpes zoster $(\mathrm{HZ})$ reactivation risk vs the general population. JAK inhibition is associated with increased infection incidence, including $\mathrm{HZ} .{ }^{4}$

Objectives: To assess long-term safety of FIL across the global clinical program with respect to $\mathrm{HZ}$.

Methods: Pts meeting 2010 ACR/EULAR RA criteria in a pooled analysis of P2 DARWIN 1-2 (D1-2), P3 FINCH 1-3 (F1-3), and long-term extension studies (D3, F4) were included. Placebo (PBO)-controlled as-randomised analysis included pts receiving FIL $100 \mathrm{mg}$ (FIL100), FIL $200 \mathrm{mg}$ (FIL200), or PBO up to week (W) 12 (D1-2, F1-2); active-controlled as-randomised analysis included pts receiving FIL100, FIL200, adalimumab (ADA), or methotrexate (MTX) up to W52 (F1, F3). Long-term as-treated analysis included pts in all 7 studies receiving FIL100, FIL200, ADA, MTX, or PBO; data after re-randomisation were included and contributed to treatment received. Exposure-adjusted incidence rates (EAIR)/100 patient-years, calculated up to the last follow-up time or day, and differences with $95 \%$ confidence intervals (Cls) were calculated from the Poisson model. Logistic regression model was used for treatment-emergent (TE) HZ risk factor analysis and odds ratio $(95 \% \mathrm{Cl})$ and $P$ value were provided.

Results: Table 1 shows TE HZ EAIRs in a pooled analysis. Rates of $\mathrm{HZ}$ were lower for FIL200 vs PBO during the 12W PBO-controlled period. At 52W, HZ rates were higher for FIL200/100 vs active control. Long-term HZ rates increased for FIL200 vs FIL100.

Table 1. EAIR of treatment-emergent herpes zoster

\begin{tabular}{|c|c|c|c|c|}
\hline & $\mathbf{N}$ & $\begin{array}{l}\text { Patient-years } \\
\text { exposure }\end{array}$ & $\begin{array}{c}\text { EAIR } \\
(95 \% \mathrm{Cl})\end{array}$ & $\begin{array}{c}\text { EAIR diff } \\
\text { (95\% } \mathrm{Cl} \text { vs PBO/active control) }\end{array}$ \\
\hline \multicolumn{5}{|c|}{ 12W PBO-controlled } \\
\hline FIL200 & 777 & 179.8 & $0.6(0.1,3.9)$ & $-0.56(-2.5,1.3)$ \\
\hline FIL100 & 788 & 181.6 & $1.1(0.3,4.4)$ & $-0.02(-2.2,2.2)$ \\
\hline PBO & 781 & 178.4 & $1.1(0.3,4.5)$ & \\
\hline \multicolumn{5}{|c|}{ Active-controlled, as-randomised ${ }^{a}$} \\
\hline FIL200 & 475 & 439.7 & $1.4(0.6,3.0)$ & $0.69(-0.7,2.1)$ \\
\hline FIL100 & 480 & 443.4 & $0.9(0.3,2.4)$ & $0.23(-1.1,1.5)$ \\
\hline ADA & 325 & 297.6 & $0.7(0.2,2.7)$ & \\
\hline \multicolumn{5}{|c|}{ Active-controlled, as-randomised ${ }^{a}$} \\
\hline FIL200 & 626 & 578.0 & $1.7(0.9,3.2)$ & $0.65(-0.8,2.2)$ \\
\hline FIL100 & 207 & 195.0 & $1.5(0.5,4.8)$ & $0.46(-1.6,2.5)$ \\
\hline MTX & 416 & 372.2 & $1.1(0.4,2.9)$ & \\
\hline \multicolumn{5}{|c|}{ Long-term as-treated ${ }^{\mathrm{b}}$} \\
\hline FIL200 & 2267 & 4047.7 & $1.8(1.4,2.3)$ & $\mathrm{NC}$ \\
\hline FIL100 & 1647 & 2032.9 & $1.1(0.8,1.7)$ & NC \\
\hline
\end{tabular}

ap to W52. 'data cut for LTE FINCH 4, Sept 19, 2019; DARWIN 3, April 26 2019.ADA, adalimumab; $\mathrm{Cl}$, confidence interval; EAIR, exposure-adjusted incidence rate; FIL, filgotinib; MTX methotrexate; NC, not calculated; PBO, placebo; $W$ week.

Figure 1 shows multivariate logistic regression model of TE risk factors.

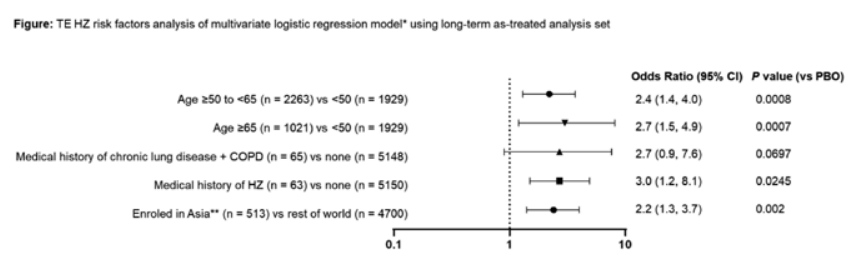

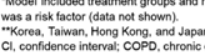

Of 104 pts with TE HZ in long-term as-treated analysis set, 5 receiving FIL200 had history of $\mathrm{HZ}$; EAIR $(95 \% \mathrm{Cl})$ was $8.7(3.6-21.0)$. Of 8 pts with multiple events, 3 had events of differing severity for the same $\mathrm{HZ}$ episode.

EAIRs (95\% CI) of TE HZ in Asia were: 3.7 (1.7-8.1) FIL200, $n=197 ; 2.8$ (1.3-6.3) FIL100, $n=158 ; 0$ ADA, $n=40 ; 2.8$ (0.4-19.6) MTX, $n=43$; and 3.4 (0.5-23.8) PBO $\mathrm{n}=77$ in long-term as-treated population. EAIRs $(95 \% \mathrm{Cl})$ in rest of the world were: 1.6 (1.2-2.1) FIL200, $\mathrm{n}=2070 ; 0.9(0.6-1.5)$ FIL100, $\mathrm{n}=1489 ; 0.8(0.2-3.1)$ ADA, $n=285$; 0.9 (0.3-2.9) MTX, n=373; and 0.7 (0.2-2.9) PBO, $n=704$ for all pts as-treated. 